\title{
The Morgan Prize for Outstanding Research by an Undergraduate Turns Twenty
}

Joseph A. Gallian

The twentieth AMS-MAA-SIAM Frank and Brennie Morgan Prize for Outstanding Research in Mathematics by an Undergraduate Student was awarded at the Joint Mathematics Meetings in San Antonio in January 2015 to Harvard graduate Levent Alpoge. In the previous six months Morgan Prize winner Manjul Bhargava received the Fields Medal, and Morgan Prize winner Jacob Lurie won one of the inaugural US $\$ 3,000,000$ Breakthrough in Mathematics Prizes and a MacArthur ("genius") Grant. In view of the awards to Bhargava and Lurie, a brief overview of the career paths of the twenty Morgan Prize winners is of interest.

1995: Kannan Soundararajan is professor and director of the Mathematics Research Center at Stanford. He obtained a bachelor's degree at Michigan and a PhD at Princeton in 1998 under Peter Sarnak. His honors include an American Institute of Mathematics Five-Year Post Doctoral Fellowship, the 2003 Salem Prize, the 2005 SASTRA Ramanujan Prize, the 2011 Infosys Prize, and an invited address at the 2010 International Congress of Mathematicians.

Joseph A. Gallian is professor of mathematics at the University of Minnesota Duluth. His email address is jga11ian@ d. umn. edu.

DOI: http://dx.doi.org/10.1090/noti1224
1996: Manjul Bhargava is the R. Brandon Fradd Professor of Mathematics at Princeton University and the Stieltjes Professor of Number Theory at Leiden University. He earned a bachelor's degree from Harvard and a PhD degree from Princeton in 2001 under Andrew Wiles. Among his awards are a Clay Mathematics Institute Five-Year Long-Term Prize Fellowship, a Clay Research Award in 2005, the 2005 SASTRA Ramanujan Prize, the 2008 AMS Cole Prize in number theory, the

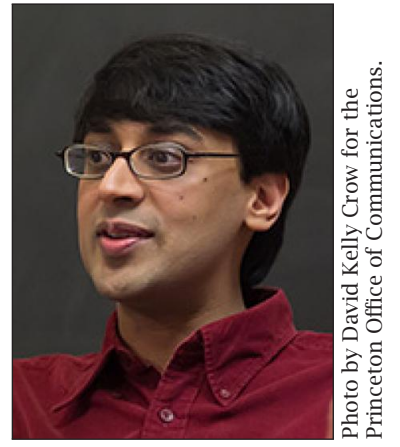

Manjul Bhargava 2011 Fermat Prize, the 2012 Infosys Prize, and a Fields Medal in 2014.

1998 ${ }^{1}$ : Jade Vinson has been at Renaissance Technologies since 2006, where he develops automated trading of financial instruments using mathematical and statistical methods and risk management. He obtained a bachelor's degree at Washington University and a PhD at Princeton in 2001 under Peter Sarnak. He held positions at the Whitehead Institute in 2001-2004 and at the Broad Institute in 2004-2006. Vinson is a member of the

\footnotetext{
${ }^{1}$ For the first two prizes the designated year of the prize was the year the nomination was due. Starting in 1998 the designated year was the year the prize was given. So there was no gap in 1997.
} 
Board of Trustees of the Museum of Mathematics in New York and has published papers in combinatorics, discrete geometry, complex analysis, measure theory, number theory, potential theory, and genomics.

1998: Daniel Biss is a member of the Illinois Senate. He received a bachelor's degree from Harvard and a PhD from MIT in 2002 under Michael Hopkins. He was a Clay Five-Year Long-Term Research Fellow, a visiting scholar at the Institute for Advanced Study, and an assistant professor at the University of Chicago. He served in the Illinois House of Representatives from 2011 to 2013 and received 66 percent of the vote in the 2012 Illinois election for the senate.

2000: Sean McLaughlin is a software engineer at Engineers Gate LLP in Brooklyn, New York. He received a bachelor's degree from the University of Michigan and a master's degree in computer science from Carnegie Mellon University. He has held positions at Jane Street Capital and Google. McLaughlin has published five papers in the areas of discrete geometry, computer science, and logic.

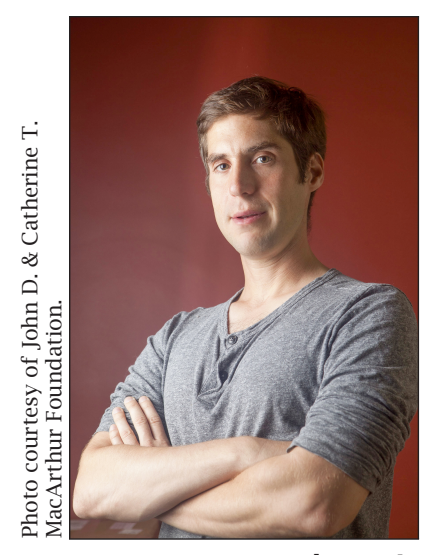

2001: Jacob Lurie is a professor at Harvard. He received a bachelor's degree from Harvard and a PhD from MIT in 2004 under the supervision of Michael Hopkins. He held a postdoctoral fellowship at Harvard from 2004 to 2007, was associate professor at MIT in 2007-2009, and became a professor at Harvard in 2009. Lurie was one of the inaugural winners of the US $\$ 3,000,000$ Breakthrough Prize in Mathematics in 2014 and received a MacArthur "Genius Grant” Fellowship in 2014. Jacob Lurie Much of his research appears in two major treatises: Higher Topos Theory and Higher Algebra.

2002: Ciprian Manolescu is a professor at UCLA. He received a bachelor's degree from Harvard and a PhD from Harvard in 2004, supervised by Peter Kronheimer. He has been a Clay Research Fellow, a postdoctoral fellow at Princeton, and an assistant professor at Columbia before moving to UCLA in 2008. He received the European Mathematical Society Prize in 2012 and the Robert Sorgenfrey Distinguished Teaching Award at UCLA in 2011. Manolescu has published papers in Annals of Mathematics, Duke Mathematical Journal, and Mathematische Annalen.

2003: Joshua Greene is an assistant professor at Boston College. He received a bachelor's degree from Harvey Mudd and a $\mathrm{PhD}$ from Princeton in 2009 under Zoltán Szabó. He has held an NSF Graduate Research Fellowship and an Alfred P.
Sloan Foundation Research Fellowship. He was an NSF Postdoctoral Research Fellow at Columbia University from 2009-2011 and has published papers in Annals of Mathematics, the American Journal of Mathematics, Inventiones Mathematicae, Advances in Mathematics, and the Bulletin of the London Mathematical Society.

2004: Melanie Wood is an assistant professor at the University of Wisconsin-Madison. She received a bachelor's degree from Duke and a PhD from Princeton in 2009 under Manjul Bhargava. She has received a Gates Cambridge Scholarship, a Fulbright fellowship, an NSF graduate fellowship, and an American Institute of Mathematics FiveYear Fellowship. Wood was a Szegő Assistant Professor at Stanford from 2009-2011. She has given invited addresses at the MathFest of the Mathematical Association of America and at the Joint Mathematics Meetings; she also gave a Pi Mu Epsilon J. Sutherland Frame lecture. Wood has published over fifteen papers in number theory.

2005: Reid Barton is a PhD candidate at Harvard. He received a bachelor's degree from MIT. He is a consultant for Dropbox and was employed at Ksplice.

2006: Jacob Fox is a professor at Stanford. He received a bachelor's degree from MIT and a $\mathrm{PhD}$ from Princeton in 2010 under Benny Sudakov. His work is supported by a Packard Fellowship, an NSF CAREER award, an Alfred P. Sloan Research Fellowship. He was previously supported by a Simons Foundation Fellowship and an MIT NEC Corporation grant. He received the SIAM Discrete Mathematics Dénes König Prize in 2010 and an Edmund F. Kelly Research Award from MIT in 2012. Fox has over sixty-five publications with thirtyseven coauthors in journals such as Annals of Mathematics, Journal of the American Mathematical Society, SIAM Journal of Discrete Mathematics, Advances in Mathematics and Duke Mathematical Journal. He has given over eighty-five invited talks, among them was one at the 2014 International Congress of Mathematicians.

2007: Daniel Kane is an assistant professor with a joint appointment in mathematics and computer science at UC San Diego. He obtained a bachelor's degree from MIT and a $\mathrm{PhD}$ from Harvard in 2011 under Barry Mazur, Benedict Gross, and Henry Cohn. He was an NSF Postdoctoral Fellow at Stanford from 2011-2014. Kane has published over fifty papers with forty-seven coauthors in mathematics and computer science.

2008: Nathan Kaplan is a Gibbs Assistant Professor at Yale. He received a bachelor's degree from Princeton and a PhD from Harvard in 2013 under Noam Elkies. Kaplan has published twelve articles on number theory, semigroups, and monoids. He 
has appeared on the television game shows "Who Wants to Be a Millionaire" and "Million Second Quiz."

2009: Aaron Pixton holds a postdoctoral position at Harvard supported by a Clay Mathematics Institute Five-Year Long-Term Prize Fellowship. He received a bachelor's degree from Princeton and a PhD from Princeton in 2013 under Rahul Pandharipande. Pixton has published ten papers in algebraic geometry and number theory.

2010: Scott Kominers is a Junior Fellow at the Harvard Society of Fellows, a research scientist at the Harvard Program for Evolutionary Dynamics, and an associate of the Harvard Center for Research on Computation and Society. He has a bachelor's degree from Harvard and received a $\mathrm{PhD}$ in business economics from Harvard in 2011. From 2011 until 2013, he was the inaugural Research Scholar at the Becker Friedman Institute for Research in Economics at the University of Chicago. Kominers has published twenty-five papers in areas such as number theory, game theory, economics, social and behavioral sciences, and discrete geometry.

2011: Maria Monks is a $\mathrm{PhD}$ candidate and Hertz Foundation Fellow at UC Berkeley specializing in algebraic combinatorics. She received a bachelor's degree from MIT and has published six papers in the areas of discrete mathematics, combinatorics, and number theory.

2012: John Pardon is a PhD candidate at Stanford. He has eight publications in the areas of manifolds and cell complexes, probability theory, and algebraic geometry. His papers have appeared in journals such as Annals of Mathematics, Annals of Probability, and the Journal of the American Mathematical Society.

2013: Fan Wei is a $\mathrm{PhD}$ candidate at Harvard. She received a bachelor's degree from MIT and has worked at Microsoft and GE Energy. Wei has three publications in combinatorics.

2014: Eric Larson is a $\mathrm{PhD}$ candidate and Hertz Foundation Fellow at MIT. He received a bachelor's degree from Harvard and has published six papers in the areas of algebraic geometry and number theory.

2015: Levent Alpoge is a Churchill Scholar at the University of Cambridge. He received a bachelor's degree from Harvard and will attend graduate school at Princeton in 2015. Alpoge has published six papers in number theory and combinatorics.

Harvard has had eight Morgan Prize winners, followed by MIT with four and Princeton with three. For graduate schools chosen by the Morgan Prize recipients, Princeton leads with eight, followed by Harvard with six and MIT with three.

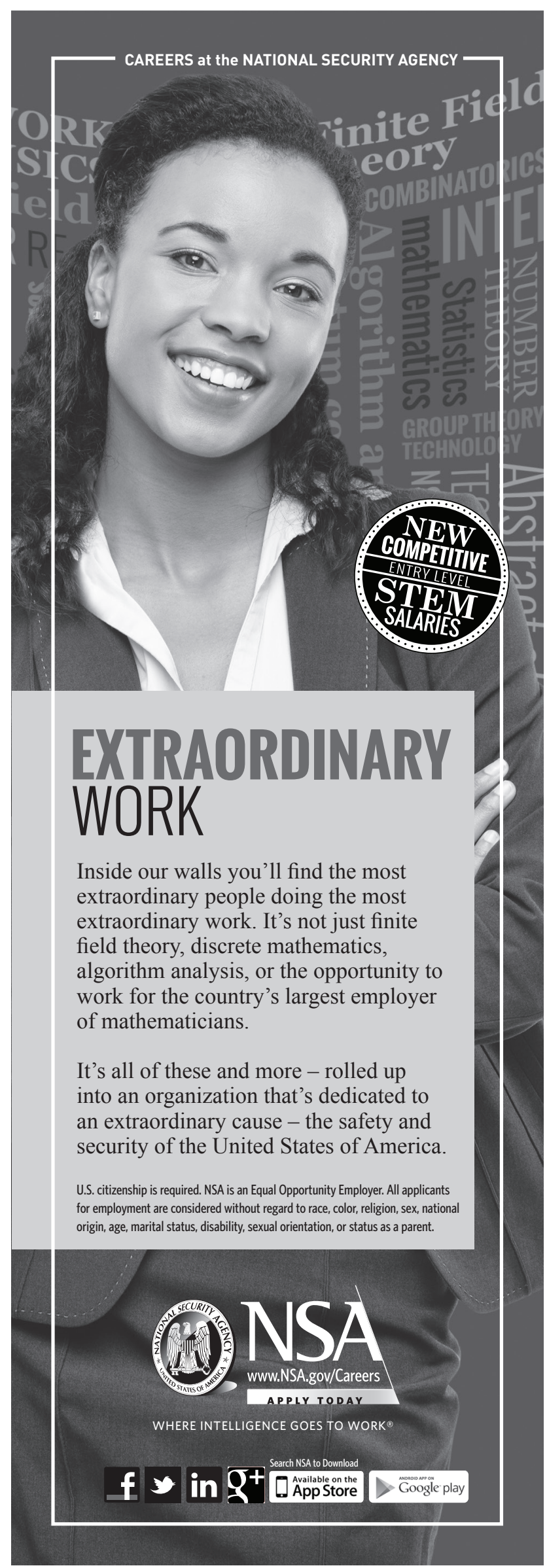

